\title{
Extraction of unexpectedly retained wire after endobronchial ultrasound
}

\author{
Raisa Bushra, MBBS, ${ }^{a}$ Navid Ahmadi, MD, BSc, ${ }^{a}$ Saman Pradeep, MBBS, MRCS, MD, ${ }^{b}$ \\ Safwat Hamad, MBBS, FRCP, ${ }^{c}$ and Aman Coonar, BSc, MBBS, MD, MRCP, FRCS CTh, ${ }^{a}$ Cambridge, \\ London, and Stevenage, United Kingdom
}

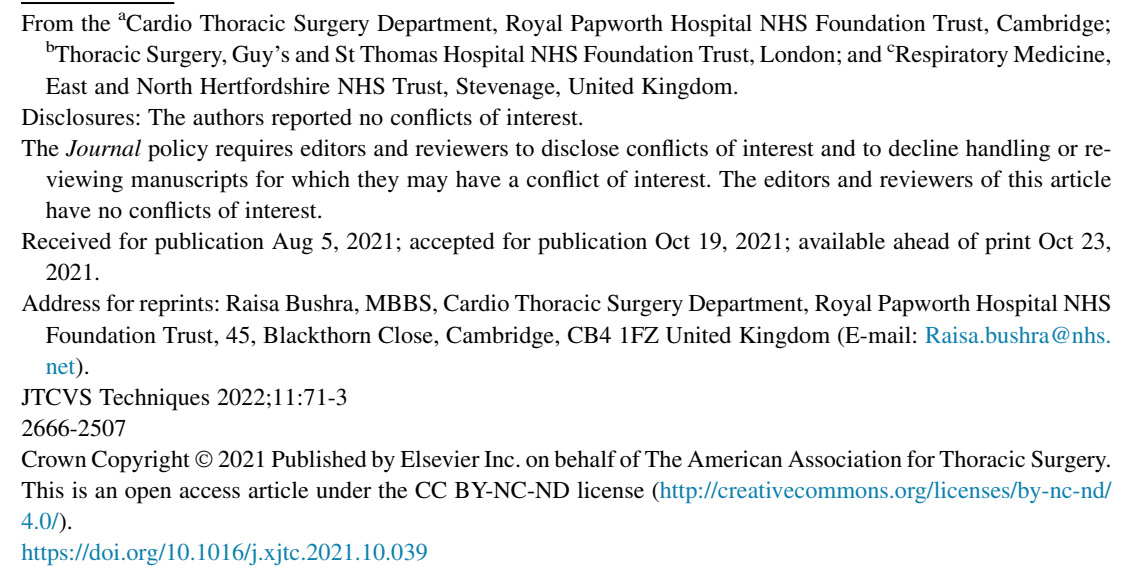

Video clip is available online.

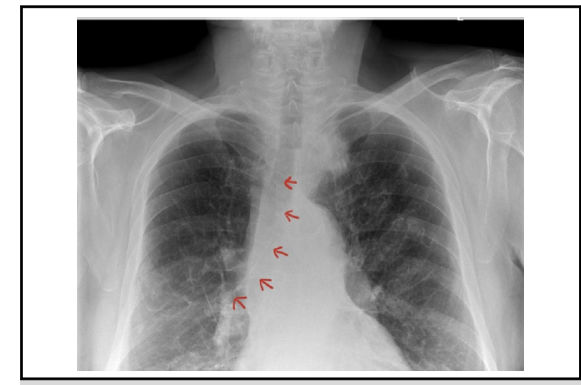

CXR showing 10-cm long broken wire from midtrachea to the right main bronchus.

\section{CENTRAL MESSAGE \\ This is an unusual case of a re- tained foreign body following EBUS and biopsy. Human factors, product failure, and the impor- tance of using periprocedure checklists are the main lessons.}

See Commentary on page 74 .
Endobronchial ultrasound (EBUS) is an established, lessinvasive technique that combines bronchoscopy with ultrasonography for sampling lymph nodes and has an important role in the assessment of lung cancer. EBUS is relatively inexpensive with a low risk of complications. ${ }^{1}$

EBUS can be performed with the patient under general anesthesia or as conscious sedation. To minimize cough, $1 \%$ or $2 \%$ lidocaine is given. First, a flexible bronchoscope is used for the initial airway examination, followed by an EBUS scope. Although the sonographic appearance can suggest malignancy, selective transbronchial needle aspiration (TBNA) is used to collect a biopsy. The biopsy needle has 2 parts: (1) the needle and (2) the inner stylet, which is a long wire that runs through the middle of the needle (Figure 1). The procedure involves real-time ultrasoundguided passage of the needle with a wire through the bronchoscope. After targeting the lymph node, the inner wire is removed from the needle to aspirate samples and when finished the wire is reintroduced in the needle to push sample material out and into the target to avoid reseeding of the tissue. Finally, the scope is removed after taking adequate samples.
EBUS is considered a safe procedure, and complications are rare. The most common complications reported are hemorrhage, infection, pneumothorax, airway injury, respiratory failure, and hypotension. ${ }^{2}$ Here, we present an unusual case of a retained wire following an EBUS procedure.

\section{CASE REPORT}

An 83-year-old man with a history of chronic obstructive pulmonary disease, myocardial infarction with coronary stent, and 5-cm abdominal aortic aneurysm was admitted for day-case EBUS TBNA for a cT2bN3M0 left upper lobe non-small cell lung carcinoma. In this case, a 21-G needle and 900-mm wire were used to take samples from lymph node stations. The procedure appeared uneventful, and the patient was discharged the same day. When he attended to receive results, he reported a persistent cough and new mild recurrent hemoptysis. A radiograph of the chest showed a foreign body (FB) (Figure 2). A computed tomography scan performed urgently showed a wire in the midtrachea extending through the anterior wall of the 


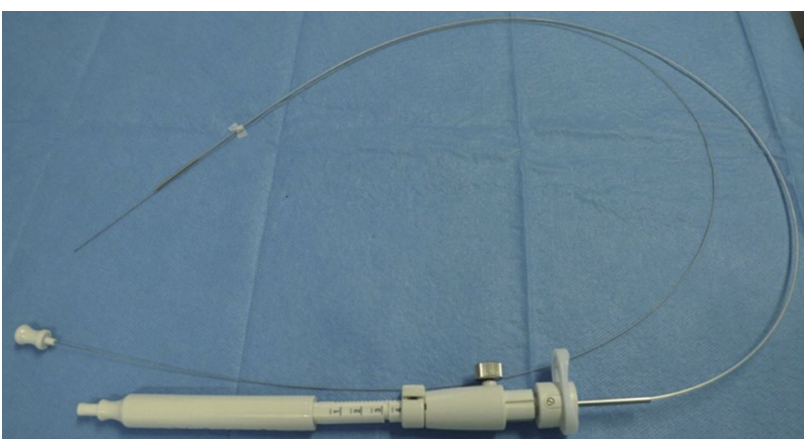

FIGURE 1. The endobronchial ultrasound needle and the inner wire.

trachea at the right bronchial bifurcation and entering the right upper lobe pulmonary artery (Figures 3 and 4, Video 1) It was suspected that the metallic FB was the wire from the EBUS TBNA.

The patient was urgently referred to our center. With the patient under general anesthesia, the airway was inspected by flexible bronchoscopy, which showed an approximately $10-\mathrm{cm}$ narrow metal wire in the airway, with the proximal end approximately $5 \mathrm{~cm}$ below the vocal cords and piercing the tracheal wall just above the origin of the right main bronchus. It appeared that the FB was the broken wire from EBUS TBNA. Extracting the FB via flexible bronchoscopy (Video 2) was not possible, as the small-caliber, flexible instruments could not retain a grip; however, it was removed easily via a rigid bronchoscopy and rigid forceps. Visualization of the puncture site showed no notable bleeding, and the patient remained stable.

The main potential risks were further injury to the pulmonary artery, further migration or embolization, inability to remove the wire, or bleeding into the airway. Instruments were on standby to perform open surgery. The extraction procedure was indeed uneventful, and there were no

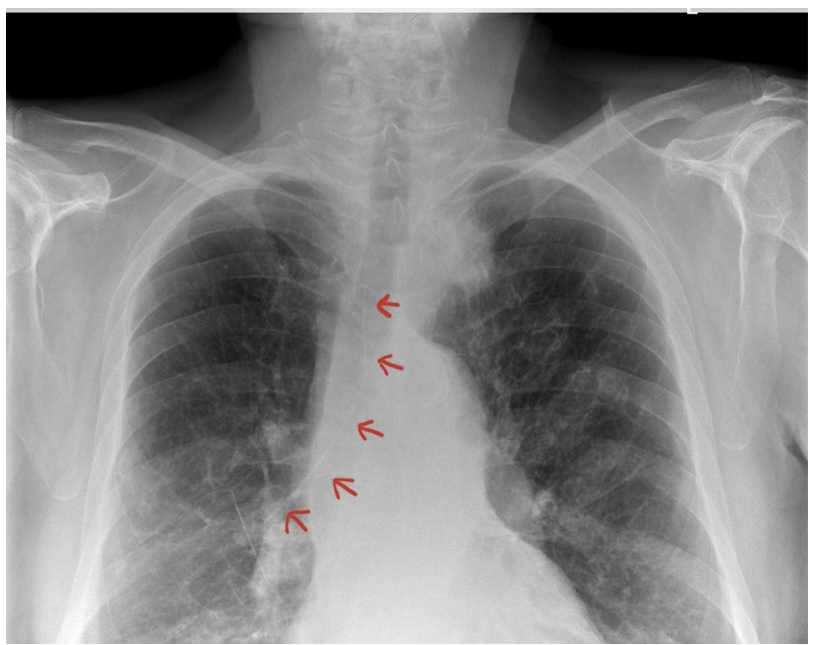

FIGURE 2. Radiography of the chest showing $10-\mathrm{cm}$ broken wire from midtrachea to the right bronchus.

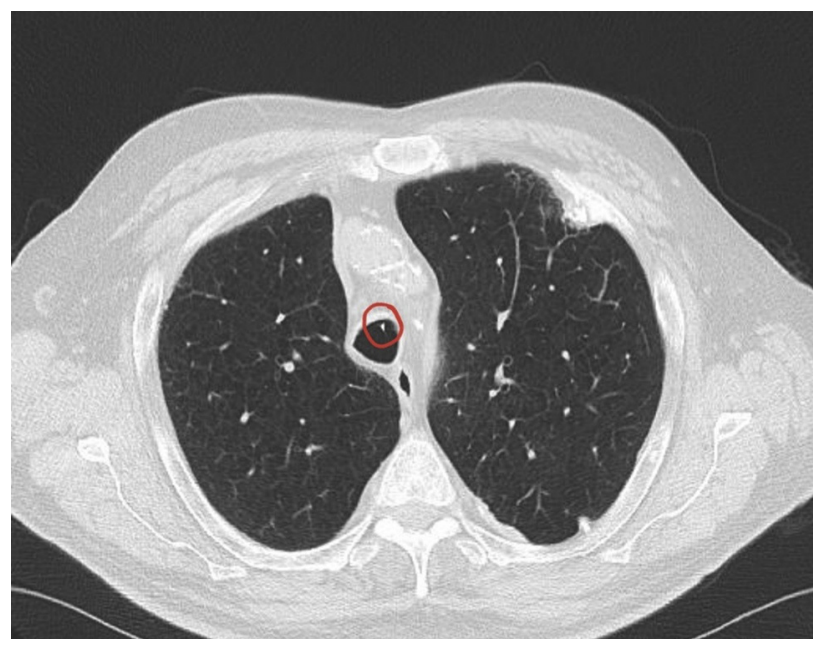

FIGURE 3. Computed tomography scan showing a narrow-caliber wire in the trachea.

immediate complications in the postoperative period. Subsequent radiographs of the chest showed no pneumothorax or collection. The patient was discharged on antibiotics for 7 days.

\section{DISCUSSION}

EBUS is established for the diagnosis and staging of various lung malignancies and evaluation of unknown mediastinal lesions. ${ }^{3}$ According to studies, the rate of complications from EBUS is less than 1\% with needle breakage is $0.2 \% .{ }^{4}$ In this case, the wire was inner stylet of the EBUS needle probably broke during the removal of the scope after taking samples.

Various factors could contribute to this retained wire. This could relate to the manufactured item or to the detail of the procedure. In a review of 9830 surgical procedures,

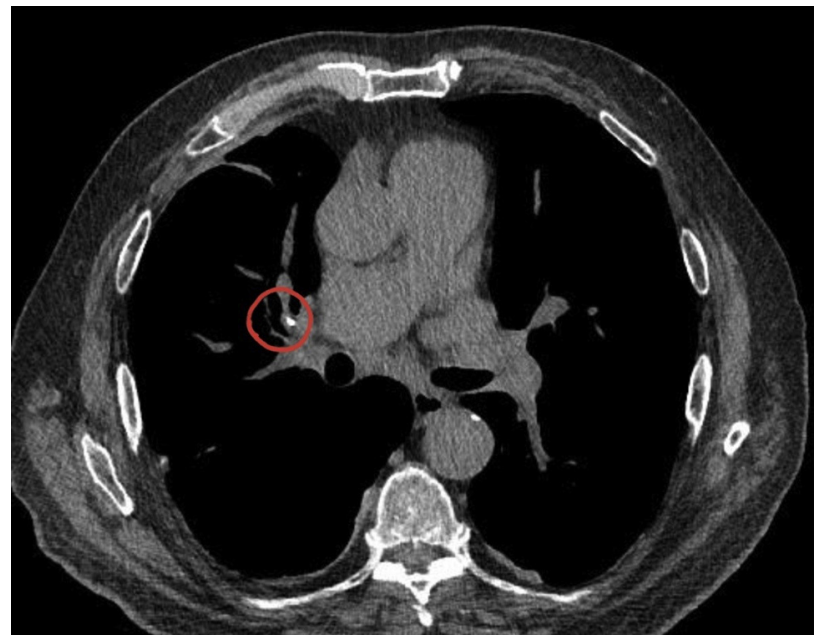

FIGURE 4. Computed tomography scan showing wire into the right upper lobe pulmonary artery. 


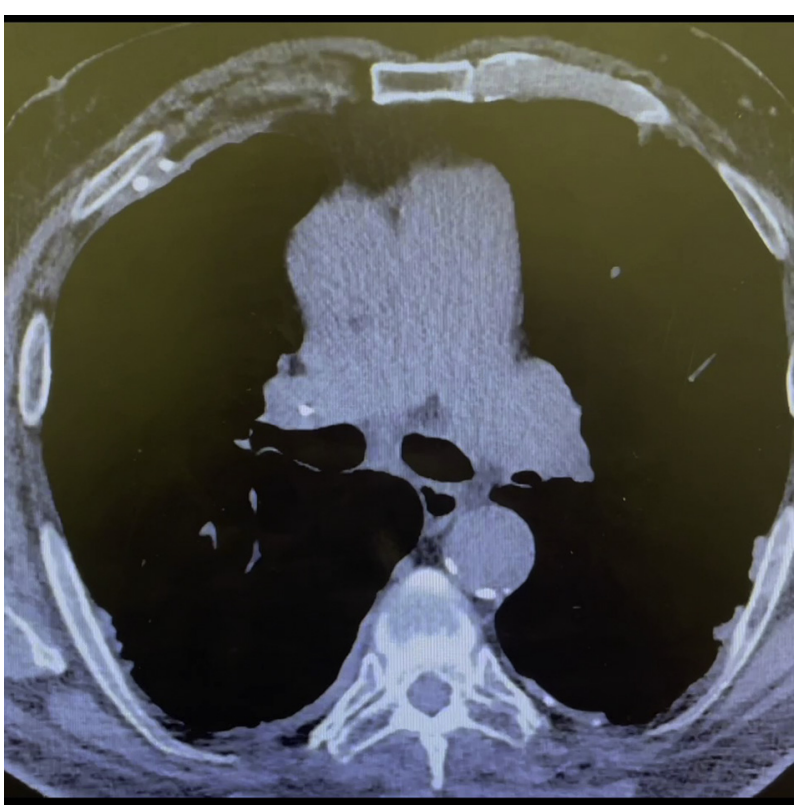

VIDEO 1. Computed tomography scan showing extent of wire in the chest from midtrachea extending through the right bronchial bifurcation and entering the right upper lobe pulmonary artery. Video available at: https://www.jtcvs.org/article/S2666-2507(21)00730-6/fulltext.

complications occurred in 332 patients, and approximately $50 \%$ were attributed to human factors. ${ }^{5}$

Perioperative checks and formal sign out are important in the prevention of inadvertently leaving items. This process should include careful inspection of all parts of the equipment, including the length of the wire and the tip of the needle. In addition, a formally reported postprocedure radiograph of the chest could be considered to look for any retained FB or other complications.

In case of accidental needle or wire breakage, it is usually removed during the original procedure. However, in this case, we report an unusual case of accidental wire breakage during an EBUS TBNA procedure that was not detected during the procedure and was removed after 24 days.

Radiographs of the chest and computed tomography scans were performed following an EBUS procedure to diagnose and locate the exact position of the broken wire. In terms of treatment, rigid bronchoscopy has been the

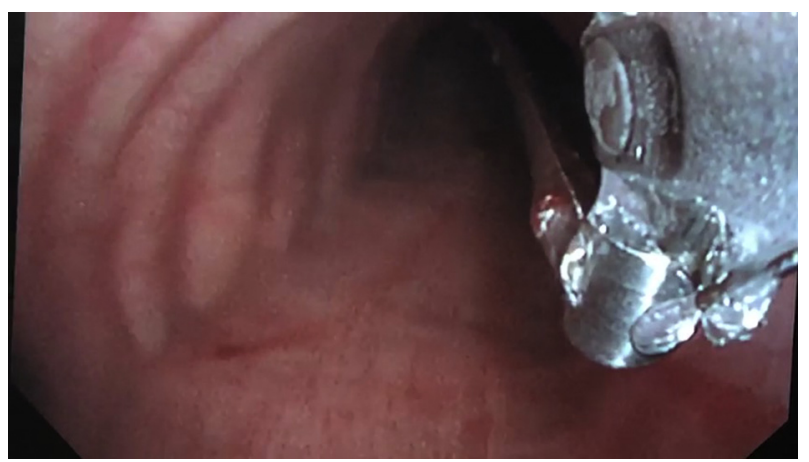

VIDEO 2. Shown is the extraction procedure via flexible bronchoscope, which was not possible, as the small-caliber, flexible instruments could not retain a grip. Video available at: https://www.jtcvs.org/article/S26662507(21)00730-6/fulltext.

recommended technique for the removal of a broken needle from the trachea or bronchus. In our case, we were able to remove the broken wire successfully by rigid bronchoscopy without further complication. The patient provided informed written consent for publication.

\section{CONCLUSIONS}

A retained surgical instrument following a procedure is to be avoided. Human factors, product failure, and not adhering to periprocedure checks are the main contributors to a retained FB following a surgical procedure. Thus, we should meticulously perform postprocedure instrument checks for every intervention including after EBUS TBNA.

\section{References}

1. Navani N, Lawrence DR, Kolvekar S, Hayward M, McAsey D, Kocjan G, et al Endobronchial ultrasound-guided transbronchial needle aspiration prevents mediastinoscopies in the diagnosis of isolated mediastinal lymphadenopathy: a prospective trial. Am J Respir Crit Care Med. 2012;186:255-60.

2. Asano F, Aoe M, Ohsaki Y, Okada Y, Sasada S, Sato S, et al. Complications associated with endobronchial ultrasound-guided transbronchial needle aspiration: a nationwide survey by the Japan Society for Respiratory Endoscopy. Respir Res. 2013;14:50.

3. Darwiche K, Özkan F, Wolters C, Eisenmann S. Endobronchial ultrasound (EBUS) — an update 2017. Pneumologie. 2017;71:798-812 [in German].

4. von Bartheld MB, van Breda A, Annema JT. Complication rate of endosonography (endobronchial and endoscopic ultrasound): a systematic review. Respiration. 2014;87:343-51.

5. Fabri PJ, Zayas-Castro JL. Human error, not communication and systems, underlies surgical complications. Surgery. 2008;144:557-63; discussion 63-5. 